stresses ; the same may be said of my Eqs. (243) and (243a). Equations (1), (243) and (243a), although thermodynamically correct, are not such that their derivatives lead immediately to the desired results. Nevertheless, the relations between mechanical and electrical quantities, in the commonly accepted form of coupling equations with stresses applied externally, are obtained by appropriate treatment of these derivatives, as may be seen by inspection of my Eqs. (186), (246), (248a) and (250).

On page 246 of my book are four equations analogous to the expressions for the internal energy $U$, the Helmholtz function $A$, the Gibbs function $G$, and the enthalpy $H$. Although the analogies are not exact, the equations were formulated as leading to the defining expressions for the piezo-electric coefficients.

More recently, I have undertaken the formulation of a set of equations in the form of exact differentials, such that, when integrated, the partial differentials lead directly to expressions for the coupling between thermal, mechanical and electrical quantities. It turns out that this can be done very simply by adding to the thermal and mechanical terms in the $d U, d H$, $d A$ and $d G$ functions, further terms expressing the electrical contribution to the energy. In the equations below, the field-strength $E$ and polarization $P$ are treated as generalized force and co-ordinate, respectively. Absolute temperature and entropy are denoted by $\Theta$ and $\sigma$, while the stress and strain matrices are denoted by $T$ and $S$. With solids, $-T$ and $S$ replace the pressure $p$ and volume $v$ that are used in the case of gases or vapours; here each component of $T$ is considered as positive when it tends to produce a positive component of $S$. The equations are :

$$
\begin{aligned}
& d U=\Theta d \sigma+T d S+E d P \\
& d H=\Theta d \sigma-S d T+E d P \\
& d A=-\sigma d \Theta+T d S-P d E \\
& d G=-\sigma d \Theta-S d T-P d E
\end{aligned}
$$

From Maxwell's relations, and assuming (over a limited range) known linear relations between $\Theta$ and $\sigma$, between $T$ and $S$, and between $E$ and $P$, one can integrate and find equations for $U, H, A$ and $G$. (The extension of the thermodynamic equations to include electrical parameters was developed independently by Prof. K. S. Van Dyke and discussed by him in "A Manual of Piezoelectric Data", Fifth Semi-Annual Report to U.S. Army Signal Corps, Part II, under Contract No. W28-003 se-1556 with Wesleyan University, January 20, 1948. I am indebted to him, and also to Dr. J. S. Mendousse, for helpful suggestions.) The partial derivatives of these equations yield at once the usual equations for coupling, in which the externally applied stress and the actual field in the crystal are given directly. These coupling equations may be regarded as equations of state. The equation for $d G$ leads to Eq. (2) in my book. The equations for $d A, d U$ and $d H$ lead, respectively, to modifications of my Eqs. (1), (243) and $(243 a)$.

It is easily shown that $d A$ as expressed above is related to $d \xi$, as obtained from my Eq. (1), as follows :

$$
d \xi=-d A+2 c E S d S .
$$

The fact that in using Eq. (1) a discrimination had to be made between internal and external piezoelectric stresses may be said to be due to the presence of the term $2 c E S d S$. If I had written Eq. (1) with the sign of the first term reversed, $d \xi$ would have been the same as $-d A$ and all stresses in the derivatives would have been external.

Equations similar to the foregoing may also be written, in which the polarization $P$ is replaced by the displacement $D$.

Wesleyan University,

Middletown, Conn. Sept. 2.

${ }^{1}$ Cook, Nature, 161, 524 (1948).

" "Piezoelectricity" (McGraw-Hill Book Co., Inc., New York, 1946). Walter G. Cady

\section{Pulsating Stars and Nuclear Energy}

THE explosion in an 'atom bomb' occurs when two masses of uranium-235, each too small to retain sufficient neutrons to start a chain reaction, are brought into juxtaposition within a certain critical volume. A similar state of affairs will account for the phenomena of pulsating stars, but with this difference, that whereas in terrestrial experiments the reacting material is dissipated by the force of the explosion, in the interior of a star those heavy nuclei which have escaped fission during the course of one explosion are reconcentrated by gravitational attraction and are available as a neutron source, or for fission, a few days later.

The data necessary for mathematical investigation have not yet been fully released, but it may be assumed that heavy nuclei will gravitate toward the centre of a star when convection currents and other types of instability have subsided. With the immeasurably larger quantities of available matter as compared with terrestrial standards, the critical conditions of explosion will be quite different. Instead of a few grams or kilograms of highly concentrated fissile material brought together into a critical volume measured in cubic centimetres, it is possible that neutron generation will exceed dissipation when the necessary minimum number of tons is concentrated into a space of thousands or millions of eubic kilometres. If the pressure of radiation engendered by nuclear fission is great enough to overcome the structural forces in the star, it will reach the surface and carry away with it a halo of gaseous material, and this is clearly seen in the case of a nova. If, however, the gravitational field and the opacity of the star are sufficient to withstand the pressure, the greater part of the explosion will be kept within the bounds of the star, and after a period of expansion and increased luminosity the star will collapse under its gravitational field, until the reactive materials have again been brought together in sufficient concentration to start another cycle.

Although mathematical proof is not yet available, this suggestion will account in general terms for the successive phases in the cycle of the Cepheid variables - the rapid expansion with little change of surface brightness, due to a wave of compression from within ; the slower collapse under gravitational force, showing irregularities due to secondary waves; the quantitative relation between absolute magnitude and period ; and the almost limitless supply of energy. In the case of supernovæ, quantitative considerations of the energy radiated support the belief that the origin of the energy is a nuclear chain reaction ${ }^{1}$.

Buckshead, Townshend,

D. Stanley-Jones 